\title{
Left atrial ball valve thrombus Treatable cause of clinical deterioration in a patient with mitral stenosis
}

\author{
R G DENT, J P R DICK, R CORY-PEARCE \\ From Papworth Hospital, Cambridgeshire
}

SUMMARY A 74 year old woman with mixed mitral valve disease presented with episodes of loss of consciousness and fainting. The attacks were noted to be closely related to posture, especially crouching, and this, with the clinical finding that the radial pulse disappeared before she lost consciousness, suggested the diagnosis of a ball valve thrombus. Cardiac catheterisation confirmed the diagnosis but was followed by a fall in blood pressure necessitating emergency surgery. The only position in which the circulation could be maintained was the right lateral, with steep head down tilt, and left femoro-femoral cardiopulmonary bypass had to be established in this position. Operation confirmed the presence of a ball valve thrombus and the patient recovered uneventfully.

Left atrial ball valve thrombi and left atrial myxomata are well recognised causes of circulatory obstruction. The former have seldom been reported: they are easily removed surgically but the diagnosis is rarely made before death.

\section{Case report}

The patient, a 74 year old housewife, had rheumatic fever as a child. In 1969 she was admitted to hospital with a right lobar pneumonia and the signs of mild mixed mitral valve disease were noted; she was in sinus rhythm and the chest radiograph showed moderate cardiac enlargement. In 1976 she suffered a right hemiplegia but recovered completely within a few days. In November 1980 she presented with a six month history of progressive congestive failure despite digoxin and diuretics, and a six week history of dizziness associated with chest pain and blackouts.

On examination she was alert with peripheral cyanosis, a small amplitude regular arterial pulse, blood pressure $110 / 70 \mathrm{mmHg}$, a venous pressure of $+8 \mathrm{~cm}, 4 \mathrm{~cm}$ hepatomegaly, and ankle oedema: she was apyrexial. There was a parasternal heave, an apical pansystolic murmur, a late opening snap, and a full length diastolic murmur. A chest radiograph showed cardiomegaly with left atrial enlargement, bilateral pleural effusions, and a prominent main pulmonary artery. Her electrocardiogram showed sinus rhythm with multiple atrial ectopic beats and digoxin effect. Full blood count was normal and erythrocyte sedmentation rate 10 .

Her episodes of loss of consciousness continued on the ward, up to 10 times daily, each beginning with the patient feeling dizzy and then rapidly losing consciousness. After 20 to 30 seconds she convulsed, and then recovered in a further 2 to 3 minutes. The episodes occurred in any position but became an invariable accompaniment to attempts to defaecate. Her congestive failure was increasingly resistant to treatment. It was thought that she was suffering from epilepsy caused by her previous cerebral infarct but her condition did not improve with anticonvulsants.

Clinical examination during an attack, with simultaneous cardiographic monitoring, showed that the first event was a gradual weakening of the radial pulse with no alteration of rate or rhythm at a time when the cardiac impulse was still forcible. The patient then became unconscious, pulseless, and convulsed, at which stage irregular weak heart sounds were heard and the electrocardiagram showed atrial fibrillation. Recovery was accompanied by a return of the peripheral circulation and sinus rhythm. The clinical diagnosis was revised to left atrial ball valve thrombus 


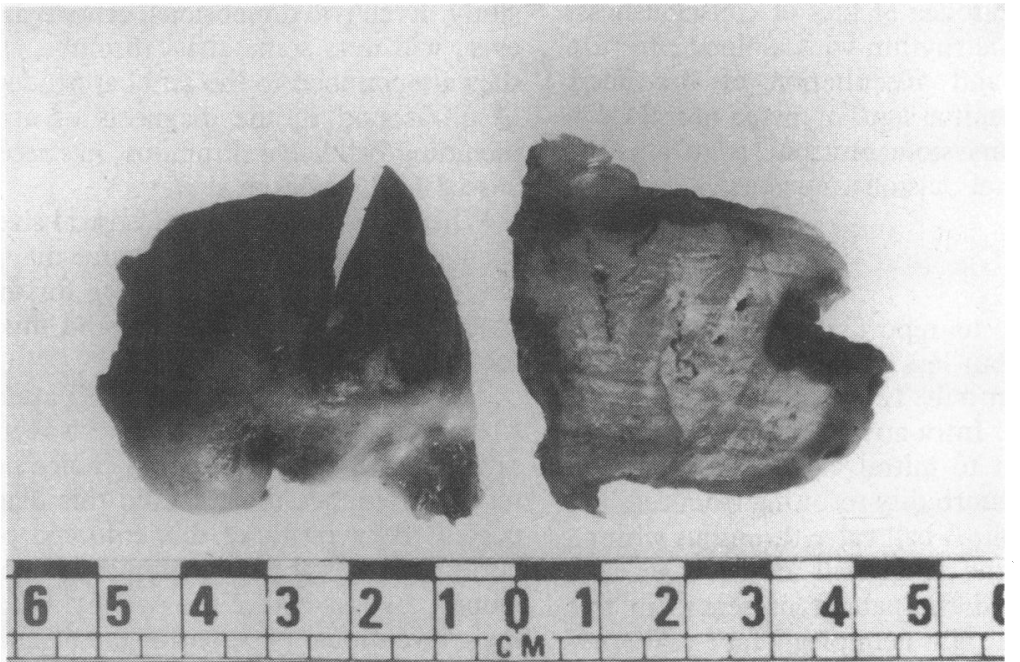

Fig. 1 Appearances of the mass removed from the left atrium bisected to show the cut surface. Note the smooth glistening outer surface. There was no attachment to the atrial wall at operation.

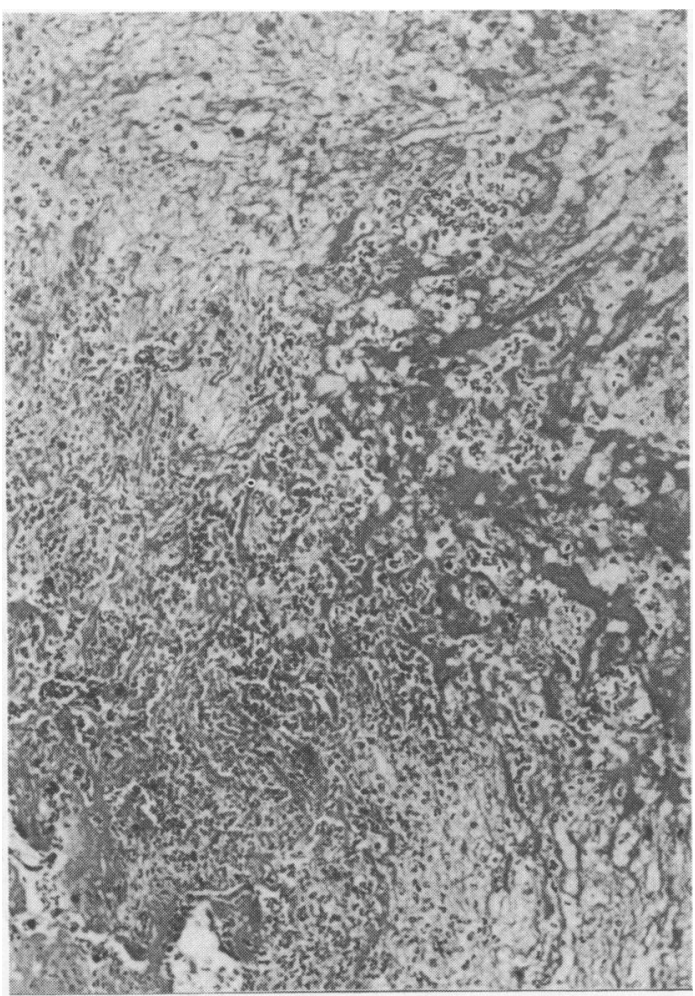

Fig. 2 Section from atrial thrombus showing red cells (pale), fibrin (grey), a few white cells (black), and a small vascular lumen of organisation (bottom left). (Haematoxylin, Phloxin, and Saffron; original magnification $\times 200$.) causing intermittent obstruction of the mitral valve orifice and she was transferred to the regional cardiothoracic unit.

M-mode echocardiography confirmed mitral stenosis but the atrial cavity was echo free. A left heart catheter confirmed a moderate mitral gradient ( 5 $\mathrm{mmHg}$ ), and a mild aortic gradient $(20 \mathrm{mmHg})$ : a pulmonary angiogram with follow through showed a large filling defect in the left atrium. The procedure was followed by a fall in the systolic arterial pressure to $40 \mathrm{mmHg}$; she was taken to the operating theatre immediately.

After induction of anaesthesia there was total circulatory obstruction in all positions except the right lateral with steep head down tilt, necessitating establishment of left femoro-femoral cardiopulmonary bypass. On full perfusion, the patient could be moved to the supine position and prepared for median sternotomy. The left atrium contained a large smooth yellowish ball 3 to $4 \mathrm{~cm}$ in diameter (Fig. 1) but was otherwise normal and there was no connection between the mass and the atrial wall. The mitral valve presented the typical appearances of fibrous, noncalcified rheumatic mitral stenosis of mild degree; the residual orifice measured $1.8 \times 1.5 \mathrm{~cm}$. There was a little subvalvar disease with shortening and fusion of the chordae tendineae. An open mitral valvotomy was performed at each commissure giving a good residual mitral orifice. Controlled filling of the left ventricle via the apical vent did not result in regurgitation. Histologically the removed mass was composed of old thrombus (Fig. 2).

Five months later the patient was well with no 
recurrence of her episodes of loss of consciousness. She remained in sinus rhythm with a blood pressure of $180 / 85 \mathrm{mmHg}$ and auscultation of the heart confirmed she had residual signs of mixed mitral valve disease with a soft pansystolic murmur, a late opening snap, and a soft mitral diastolic murmur.

\section{Discussion}

Wood was the first to report a case of ball valve thrombus in $1814^{1}$ but less than 60 cases have been reported since. ${ }^{2-4}$ In only 12 was the diagnosis suspected before death. Intra-atrial thrombus is a common accompaniment to mitral valve disease and an important source of morbidity resulting from cerebral and peripheral emboli. A ball valve thrombus with no firm attachment to the atrial wall may also act as a source of emboli, and the patient presented in this report had suffered a right hemiplegia four years previously.

The presence of a ball valve thrombus with or without a tenuous attachment to the atrial wall may in addition lead to intermittent obstruction of the mitral valve orifice with resultant syncope and sometimes more prolonged interruption of the circulation leading to death. The dramatic nature of the present patient's syncopal episodes and their relation to posture suggested the possibility of circulatory obstruction from an intracardiac mass. This was supported by the clinical findings: palpation and auscultation of the heart at the same time as palpation of the radial pulse showed that the heart continued to beat, though weakly, despite no radial pulse. Simultaneous electrocardiography showed clearly that sinus rhythm was maintained until approximately 10 seconds after the pulse was lost when atrial ectopics and then atrial fibrillation occurred, presumably as a result of cardiac ischaemia.

The differential diagnosis at this stage lay between an atrial myxoma and a ball valve thrombus. The latter was felt more likely in view of the patient's past history of rheumatic fever, the presence of typical and unchanging signs of mixed mitral valve disease for many years, and the absence of any of the other features of atrial myxoma such as fever or a raised erythrocyte sedimentation rate. M-mode echocardiography failed to show the presence of the intra-atrial thrombus which was subsequently shown at angiography and confirmed at operation. M-mode echocardiography has been recommended for the diagnosis of left atrial thrombus ${ }^{5}$ but two dimensional echocardiography has added new and more precise data to that obtained by $\mathrm{M}$-mode and has been used to detect ball valve thrombus in the atrium. ${ }^{4}$ It would have been valuable in the present case and may have avoided what proved to be a very hazardous cardiac catheter study. Even two dimensional echocardiography, how-

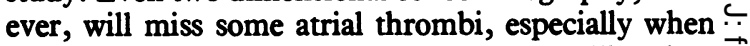
they are confined to the atrial appendage. ${ }^{4}$ The place $\overrightarrow{\vec{s}}$ of ultrasound in the diagnosis of atrial thrombus, including ball valve thrombus, has recently been dis- $\frac{C}{0}$ cussed by Donaldson et al. ${ }^{4}$

When an atrial mass is of critical size, posture and $\vec{\nabla}$ changes in intrathoracic pressure may prove impor- $\frac{2}{0}$ tant determinants of the liability for the mass to ob- $\infty$ struct the mitral valve orifice, and this was certainly $\vec{O}$ the case in our patient.

The manoeuvre of placing the patient in the right ${ }_{\mathscr{\omega}}$ lateral steep head down position was in this case life saving and should be the first choice position during resuscitative measures, should this diagnosis be suspected. As a result of this enforced position it was necessary to establish left femoro-femoral cardiopul- 8 monary bypass before the patient could be placed in $ᄋ$ the conventional position for median sternotomy.

Most cases of atrial ball valve thrombus occur in $>$ patients with longstanding mitral valve stenosis though there is one report of a case in a patient with $\vec{\bullet}$ bacterial endocarditis and profuse vegetations. ${ }^{6} \mathrm{~A}$ past history of peripheral or cerebral emboli is common and the patients are usually in established atrial fibrillation. Clinical evidence of persistent congestive cardiac failure and poor peripheral circulation is often found but may simply reflect severe mitral stenosis. Once the diagnosis is established by echocardiography $\cong$ or angiography, removal is curative and may be com- $\overrightarrow{\overrightarrow{0}}$ bined with valvotomy or valve replacement if appro- 3 priate.

We would like to thank Dr K D Allanby and Dr M C Petch for permission to report this case.

\section{References}

1 Wood W. Letter enclosing the history and dissection of a case, in which a foreign body was found within the heart. $\frac{\rho}{2}$ Edinb Med Surg f 1814; 10: 50-4.

2 Abramson JL. Ball thrombi of the heart. Ann Clin Med $\frac{\mathrm{T}}{3}$ 1924; 3: 327-64.

3 Evans W, Benson R. Mass thrombus of the left auricle. Oั Br Heart f 1948; 10: 39-47.

4 Donaldson RM, Ballester M, Olsen E. Diagnosis of atrial N thrombi by ultrasound. Thorax 1982; 37: 507-11.

5 Schweizer P, Bardos P, Erbel R, et al. Detection of left atrial thrombi by echocardiography. Br Heart $\mathcal{F} 1981 ; 45: \stackrel{0}{C}$

6 Radding RS. Ball thrombus of the right auricle. $A m \mathcal{I} \stackrel{\oplus}{+}$ Med 1951; 11: 653-7.

Requests for reprints to Dr R G Dent, Papworth $\frac{\rho}{\Phi}$ Hospital, Papworth Everard, Cambridgeshire CB3응 8RE. 